\title{
Proteolytic interstitial cell migration: a five-step process
}

\author{
Peter Friedl • Katarina Wolf
}

Published online: 21 January 2009

(C) The Author(s) 2009. This article is published with open access at Springerlink.com

\begin{abstract}
Cell migration is a multi-step process that leads to the actin-driven translocation of cells on or through tissue substrate. Basic steps involved in cell migration have been defined for two-dimensional haptokinetic migration which, however, does not provide physical constraints imposed by three-dimensional interstitial tissues. We here describe the process of pericellular proteolysis that leads to extracellular matrix (ECM) degradation and realignment during cell movement and integrate it into established steps of cell migration. After actin-driven leading edge protrusion (step I) and anterior formation of integrin-mediated focal interactions to the substrate (step II), ECM breakdown is focalized towards physical ECM barriers several micrometer rearward of the leading edge (step III). Actomyosinmediated cell contraction (step IV) then leads to rear-end retraction and forward sliding of cell body and nucleus so that a small tube-like matrix defect bordered by realigned ECM fibers becomes apparent (step V). Pericellular proteolysis is thus integral to the migration cycle and serves to widen ECM gaps and thereby lowers physical stress upon the cell body, which ultimately leads to aligned higher-oder ECM patterns.
\end{abstract}

P. Friedl $(\bowtie) \cdot$ K. Wolf

Department of Cell Biology, Nijmegen Center for Molecular Life Science, Radboud University Nijmegen Medical Centre, P.O. 9101, 6500 HB Nijmegen, The Netherlands e-mail: P.Friedl@ncmls.ru.nl

P. Friedl

Rudolf-Virchow Center for Experimental

Biomedicine and Department of Dermatology,

Venerology, and Allergology, University of Würzburg,

Josef-Schneider-Strasse 2,

97080 Würzburg, Germany
Keywords Cancer metastasis · Cell migration · Proteases · Integrins

\section{Introduction}

Cell migration is fundamental to cell and tissue dynamics in morphogenesis, immune defence, repair as well as cancer invasion and metastasis. Basic concepts on cell migration as a physiochemical process have been established using isolated cells during their movement across ECM-coated two-dimensional (2D) surfaces [1]. In the multicellular organism, cell migration across flat ECM substrate is present in epithelial migration i.e. during regeneration, such as epidermal wound healing [2], and the lateral movement of gut epithelium along the basement membrane [3]. Most cells in the mammalian body, however, migrate within threedimensional (3D) tissues, such as interstitial connective or neuronal tissue. 3D migration is carried out by different cell types using different shapes, kinetics and stringency of cellcell interactions and, thus, shows significant diversity [4]. In difference to migration across $2 \mathrm{D}$ substrate, flat and spreadout cell morphologies are prevented in 3D tissue whereas spherical shapes of varying length, ranging from short ellipsoid to elongated and spindle-shaped are maintained [5]. Rather than forming a flat continuous leading lamella, the leading edge protruding into $3 \mathrm{D}$ tissue contains one or few cylindrical pseudopodia with terminal filopodia that dynamically engage with ECM fibers [6-8]. Whereas cells moving across $2 \mathrm{D}$ surfaces contain stress fibres that span between focal adhesions, cells migrating in 3D tissues lack stress fibres but form a cortical cytoskeleton. Thus, dependent on whether cell migration occurs in $2 \mathrm{D}$ or $3 \mathrm{D}$ environments, cell morphology and actin-rich protrusions that engage with the ECM vary considerably. 


\section{Physics of 3D cell migration and proteolytic ECM fiber cleavage}

Whereas the basis of actomyosin-mediated protrusion and contractility is common to all migration types, their biophysical principles diverge. The prototypic mode of stromal cell migration is derived from the mesenchymal migration of fibroblasts or fibroblast-like cells [7]. Hallmarks of mesenchymal migration in 3D ECM are spindleshaped morphology and relatively slow migration ( 0.1 to $1 \mu \mathrm{m} / \mathrm{min})$ as well as focalized cell-matrix interactions towards the ECM scaffold that mediate high traction forces and the capability to proteolytically remodel the ECM structure during migration [5-7]. Besides in fibroblasts, mesenchymal migration is found in smooth muscle cells, pericytes, activated macrophages, neural crest cells, and many dedifferentiated cancer cells, including fibrosarcoma or breast cancer cells.

3D cell migration models have allowed to identify the physical and molecular components that determine interstitial migration, including physical resistance imposed by tissue structures, the presence of tissue gaps and trails, and the cellular capability to remodel ECM [9-11]. First, the protruding leading pseudopod is rich in F-actin and consists of several smaller protrusions that extend along and adhere to collagen fibers. If traction force is transmitted, collagen fibers become pulled and realigned towards the cell (Fig. 1A, empty arrowheads). Although in close contact with collagen fibers, protruding pseudopodia do not generate ECM degradation, visualized by the lack of collagen cleavage epitope (Fig. 1B, black arrowheads). Several $\mu \mathrm{m}$ further rearward, however,

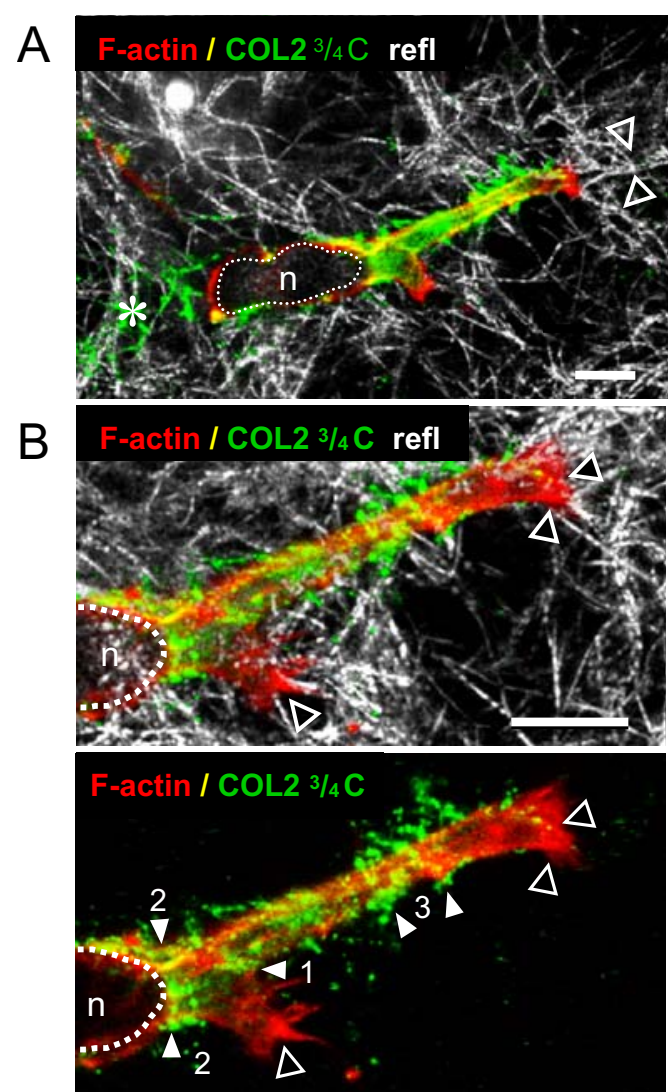

Fig. 1 Distribution of actin and pericellular proteolysis during mesenchymal migration. Overview (A) and details (B-C) of mesenchymal HT-1080 cell invading into 3D fibrillar collagen lattice. Cells were allowed to migrate within 3D fibrillar collagen lattices for $18 \mathrm{~h}$. Samples were fixed and stained with $\mathrm{COL} 23 / 4 \mathrm{C}$ Ab recognizing proteolytic neoepitope generation in collagen (green) [9] and phalloidin $(\mathrm{red})$. 3D reconstructions of confocal fluorescence and reflection signal. A Overview of mesenchymal migration. Asterisk, migration track containing partly cleaved collagen fibers. Empty arrowheads, traction of collagen fibers. B, C Leading edge segregation

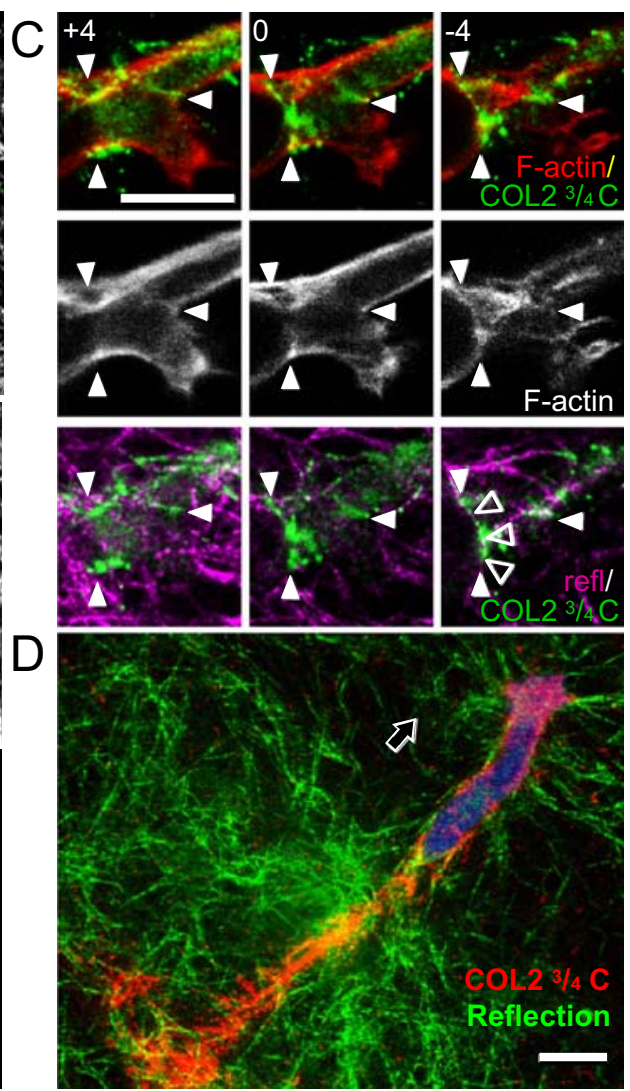

of actin-rich protrusions and zones of collagen processing. B Empty arrowheads, F-actin rich protrusions at the leading edge devoid of proteolysis. B, C White arrowheads, partly degraded belt-like collagen fibers (1) at pseudopod branches, (2) pushed by the nucleus or (3) crossing the cell body in mid-region of the cell. C Empty arrowheads, sterically impeding fiber pushed by the nucleus. Single z-sections from different z-positions (numbers, z-distance from mid section in $\mu \mathrm{m}$ ). N, nucleus (dotted line). Bars, $10 \mu \mathrm{m}$. D Proteolytic migration track, visualized by COL23/4C staining. Bar, $20 \mu \mathrm{m}$ 
collagen cleavage neoepitope is generated at cell surfacebound collagen fibers (Fig. 1b, white arrowheads). Three distinct regions of proteolytic degradation are apparent: branch points between pseudopodia (Fig. 1b, 1); curved perpendicular collagen fibers pushed forward by the nucleus (Fig. 1B, 2); and random perpendicular collagen fibers crossing the elongated cell body (Fig. 1B, 3). All these collagenolytic foci contain filamentous actin (Fig. 1C, white arrowheads) together with MT1-MMP, an important transmembrane collagenase and $\beta 1$ integrin [9]. As consequence of collagen fiber remodeling, a migration track of approximately the cell width is generated consisting of proteolytically processed collagen fibers and bundles in partly parallel alignment (Fig. 1d).

Using dynamic imaging and detection of steady-state pericellular proteolysis by the dequenching of fluorescently labeled collagen fibers, the location and temporal sequence of realignment and of collagen fiber cleavage and realignment can be detected in forward-moving cells (Fig. 2). ECM fibers crossing the cell body bear dequenched signal as consequence of proteolytic degradation (Fig. 2A). After structural fiber cleavage, the blunt ends remain bound to the cell surface and become transported forward with the moving cell body (Fig. 2B). Thereby loose fibers eventually become reoriented in parallel to the lateral cell-matrix interface, resulting in fiber alignment (Fig. 1D). Together, both static and kinetic imaging reveals the cleavage of individual collagen fibers in zones distal from the actin-rich leading edge and their subsequent realignment.

\section{Integrating proteolysis into the migration cycle: the 5-step model}

These findings allow to integrate pericellular proteolysis into cell migration as a physicochemical process in a time- and space-defined manner [1, 12] (Fig. 3a). Starting from an initial poorly polarized state within a 3D ECM structure, polarization of actin flow is initiated by different stimuli, including chemokine- and adhesion receptormediated signalling [13], resulting in the protrusion of a (leading) pseudopod (Fig. 3a, step I). If integrins engage with the substrate, cluster and form focalized adhesion complexes, the leading egde becomes anchored to the ECM and, if concurrent with cell contraction (see step IV), force is transmitted towards the ECM which consequently becomes pulled upon and realigned (Fig. 3A, step II) [14, $15]$. Because the shape of the leading edge is commonly tip-like and the diameter is only $1-3 \mu \mathrm{m}$, plasma membrane and cytoplasm flow by contact guidance along tissue structures through little gaps and tracks and therefore likely do not require ECM breakdown to protrude. Several $\mu \mathrm{m}$ further rearward, however, the cell

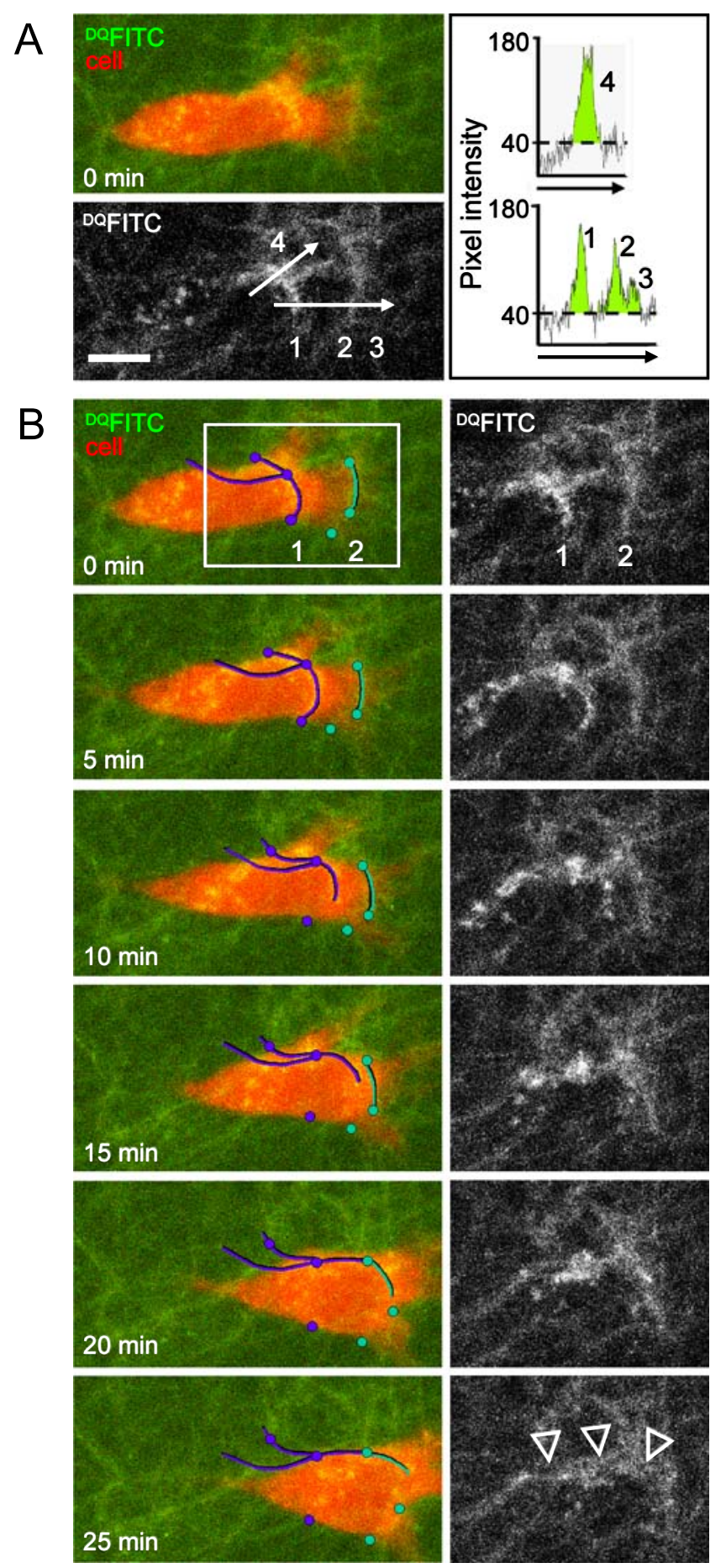

Fig. 2 Dynamic reconstruction of proteolytic collagen fiber degradation, transport and realignment during migration. Migrating HT1080 cell in 3D collagen lattice containing quenched FITC label. A Detection (left) and densitometric analysis (right, graphs) of FITC dequenching at three consecutive collagen fibers at the leading edge. B Time-series of collagen fiber cleavage (0 to $10 \mathrm{~min}$, fiber 2 ), forward transport of one blunt end (10 to $20 \mathrm{~min}$ ) and lateral alignment in parallel to the length axis of the cell (25 min, arrowheads). Fibers and stable anchor points are highlighted (left). Bar, $10 \mu \mathrm{m}$ 
Fig. 3 The five-step model of cell migration through $3 \mathrm{D}$ interstitial tissue. A Steps I-V each executing a distinct function occur in a sequential and cyclic manner and, ultimately, lead to cell migration and the structural remodeling of the ECM. White arrows: movement; red arrows: force generation. Red, f-actin; yellow cell surface proteases.

B Topographic and functional segregation of adhesion and proteolysis at the leading edge. Anterior protrusion driven by actin flow (zone I) leading to adjacent adhesion and force generation predominantly mediated by integrins (zone II) is followed by proteolytic cleavage of ECM components in a separate zone further rearward (zone III). C Time-resolved steps leading to migrationassociated pericellular proteolysis and ECM remodeling
A

non-polar, sessile state

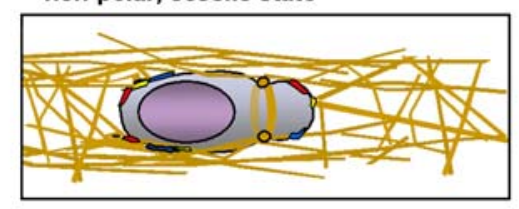

I

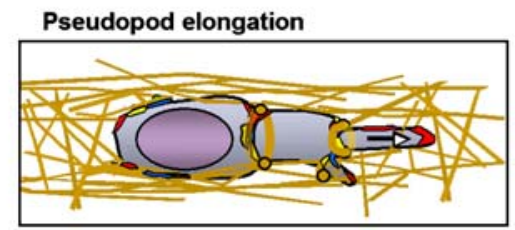

II

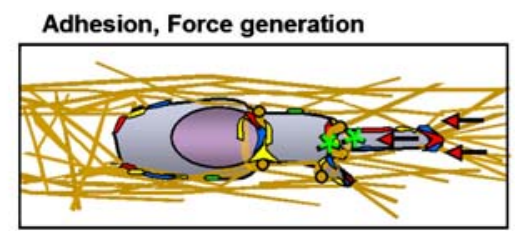

III

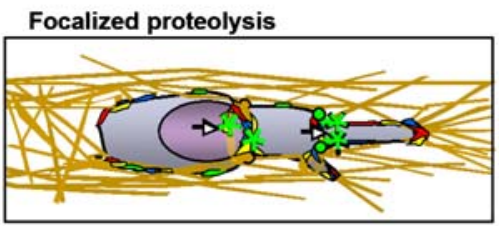

IV

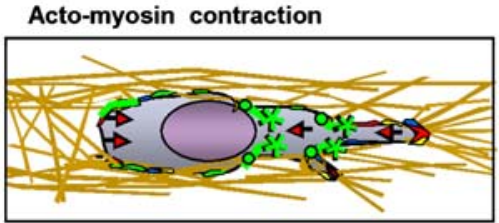

V

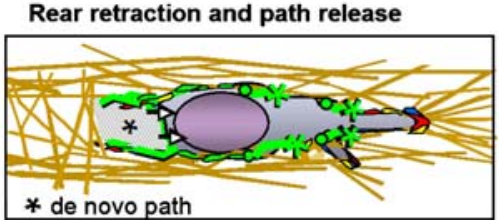

B

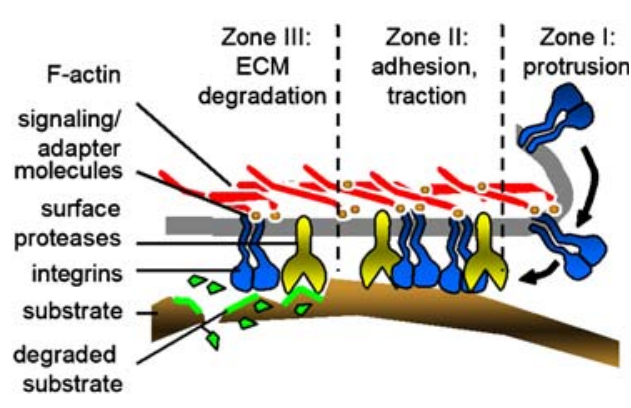

C
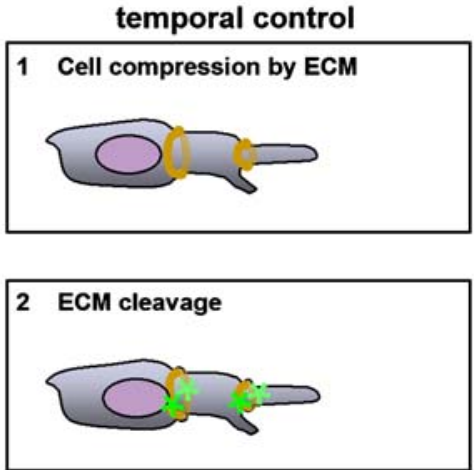

3 ECM fibre alignment

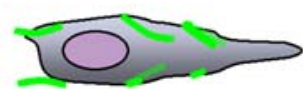

4 Matrix defect generation

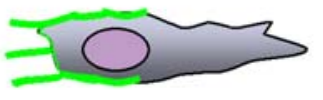

body expands with the help of the cortical actin cytoskeleton, branches, and pushes against circumferent ECM fibers that confine the cell body. Here, local proteolysis occurs together with focalized filamenous actin, clustered integrins and surface proteases [9] which leads to cleavage of fibers and local volume expansion of the cell body. Because several perpendicular fibers become cleaved in a sequential manner, sufficent space is being generated for the nucleus to slide forward along the already remodeled ECM track, rather than becoming trapped by too narrow pores (Fig. 3A, step III) [9]. Actomyosin contraction along the cellular length axis including the rear then leads to longitudinal tension
(Fig. 3A, step IV). If the leading edge attachment to the matrix outweighs that of the trailing edge, the rear end moves forward and a track of remodeled ECM becomes apparent (Fig. 3A, step V).

\section{Spatial control of pericellular proteolysis at the leading edge}

Adhesion and proteolysis are principally countercurrent processes because uncontrolled ECM degradation at or very nearby adhesion sites would compromise force generation due to the premature loss of ECM scaffold. As conse- 
quence, the cell would be surrounded by ECM fragments rather than a solid tissue structure, detach, and float in a "sea" of soluble ligand. This worst-case scenario is prevented by separating the anterior tip region from the proteolytic action, which in turn is apparent further rearward where litte or no traction force needs to be generated. Thus, adhesion and force generation, and pericellular proteolysis are executed in different subcellular regions, thereby sparing the leading edge from premature loss of substrate (Fig. 3B). The mechanisms that segregate these two zones are unknown. Likely they reside in local signalling control of proteolytic action but not in alterations of the composition of the lytic adhesion structure, because the main structural components F-actin, $B 1$ integrins and surfacelocalized MMPs are equally focalized to substrate contact points in front, mid and rear regions of the cell $[9,16]$. Further, outside pressure towards the cell body, the duration of fiber contact to the cell surface and the amount of active protease present in ECM-binding regions may be ratelimiting. Thus, fibers that either press against the cell body and/ or fibers that remain long enough in contact to the cell surface are most likely those that undergo proteolytic degradation.

\section{Temporal control of pericellular proteolysis}

The proteolytic action at the cell surface during migration is a stereotypic multi-step process (Fig. 3C). First, ECM polymer needs to physically touch the cell surface, either loosely or as compression zone which causes inward pressure upon the cell body (Fig. 3C, step 1). These very specific regions then undergo proteolytic action resulting ultimately in the focal cleavage of ECM fibers and the generation of two loose ends (Fig. 3C, step 2). As the cell moves, blunt fiber ends that continue to touch the cell surface become transported and aligned along the length axis of the cell (Fig. 3C, step 3). Possibly by secondary remodeling, such as secretion of additional ECM components, realigned fibers remain stable in their new position lateral to the cell body which becomes apparent as the rear end has released the newly formed matrix defect (Fig. 3C, step 4). The ECM defect then consists of a hollow tube the size of which approximately corresponds to the diameter of the cell. Thus, migration-associated pericellular proteolysis consists of individual biochemical and biophysical steps that ultimately lead to an oriented, compacted ECM scaffold that borders a quite linear track.

\section{Consequences of proteolytic ECM remodeling}

Proteolytic migration has consequences for both, shape and function of the moving cell and the tissue that was transmigrated. On the cell side, a linear trail is gradually formed which encompasses the cell body and limits the deformation of cell body and nucleus, thereby supporting relatively persistent forward gliding of the cell [16]. On the ECM side, randomly ordered fibrils become realigned and patterned which results in thicker bundles of largely parallel order and adjacent small tracks devoid of fibrils (Fig. 4A). Such patterns are typical for mature interstitial tissues, such as

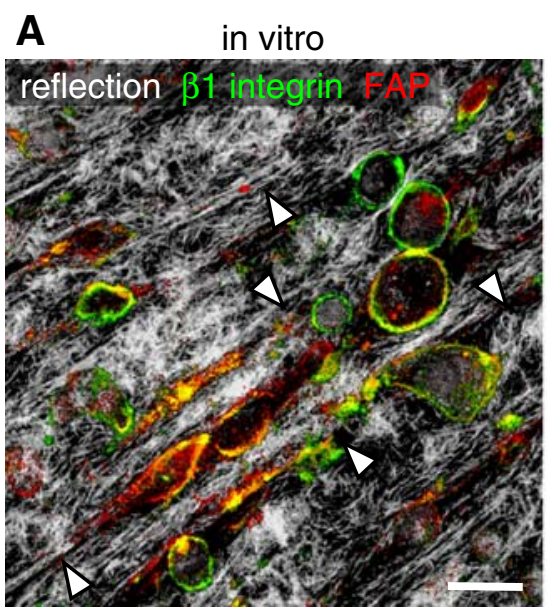

Fig. 4 Collagen-rich ECM structures in vitro and in vivo. A Alignment and condensation of collagen fibers (gray) by migrating HT1080 cells after 4 days of culture in 3D fibrillar collagen, resulting in higher-order arrangement of the ECM. Red label shows fibroblast activation protein (FAP), a serine protease with collagenolytic activity. ß1 integrins, green; collagen, gray. B Aligned dense fiber bundles and

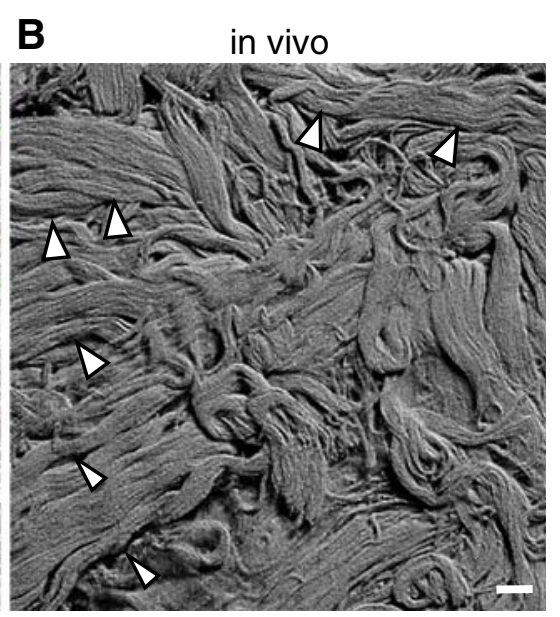

linear gaps of several micrometer in diameter in 3D human dermis, detected by multiphoton-excited second harmonic generation $(880 \mathrm{~nm})$ and topographic three-dimensional reconstruction. Arrowheads, aligned microtracks bordered by collagen fiber bundles. Bars, $20 \mu \mathrm{m}$ 
human dermis (Fig. 4B). Tissues are typically heterogeneous in structure and spacing and migrating cells preferentially migrate along constitutive tracks in loose connective tissue rather than through adjacent more compact areas. Such lowdensity tracks may serve as guidance cues for passenger cells. In inflamed tissue, leukocytes preferentially utilize trails of aligned loose connective tissue along vessels or epithelial basement membranes [17] and in cancer invasion models, these trails are filled by invading cells to form invasion strands [9]. Thus, proteolytic cell migration is a candidate mechanism to generate such ECM patterns.

\section{Deviations from the rule}

This five-step model of proteolytic migration forms an integrated program of slow migration designed to remodel tissue structure, and only mesenchymal cells are known to execute the complete 5 step-cycle. Cell migration, however, is a diverse process and different cell types employ different mechanisms, rendering the proteolytic step as optional, rather than indispensable. Cell types that do not execute step III are either cells that constitutively move without protease function, or cells after pharmacological abrogation of protease activity [18, 23].

In contrast to the here described mesenchymal migration mode, leukocytes are rapidly moving cells that employ amoeboid movement characterized by a short, ellipsoid shape. They preferentially travel through loose interstitial tissue at 10 to 40 fold higher speed than mesenchymal cells, lack focalized adhesion structures to the substrate and do not execute pericellular proteolysis while migrating [19, 20]. Instead of removing ECM barriers by proteolytic cleavage, they glide through narrow regions by flow of the cytoplasm and squeezing of the nucleus which is supported by actomyosin contraction in the cell rear [21, 22]. The zone of usually strong cell deformation through narrow tissue is a hallmark of amoeboid movement, termed constriction ring or compression zones [20, 23].

In reminiscence of leukocytes, mesenchymal cells that loose their ability to cleave ECM, can convert to nonproteolytic migration and overcome matrix barriers by squeezing through rather than degrading ECM. After treatment with protease inhibitors or RNA knockdown of MT1-MMP/MMP-14, the rate-limiting collagenase towards degradation of fibrillar collagen, invading fibrosarcoma or breast cancer cells continue to move by an amoeboid mechanism and squeeze through ECM gaps and trails, similar to leukocytes $[9,23]$. This process, termed mesenchymal-amoeboid transition, secures migration without the proteolytic step. Thus, pericellular proteolysis and ECM remodeling are secondary to cell migration and facilitate forward gliding in a persistent manner along linear tracks, yet alternative, nonproteolytic shape change and amoeboid squeezing secure a less peristent migration mode that follows preformed rather than newly remodeled tissue structures.

\section{Conclusions}

The proteolytic step is facultative in the migration cycle, dependent on the type of cell and ECM substrate. When present in a regulated manner, proteolysis prevents the cell from physical stress, retains the nucleus in round to ellipsoid state and provides a physical mechanism to align tissue structures. It further serves to establish trails of least resistance that are used by other cells for track guidance. Proteolysis is therefore a step that adds complexity to the cell migration process enabling cells to shape tissues by introducing ordered structure. On the other hand, cells that lack step III either transmigrate narrow ECM gaps by nonproteolytic strategies, such as by changing the cell morphology, or circummigrate this region by retracting the leading edge and deviating from the previous path. In conclusion, despite their clear functions in track generation and tissue remodeling, proteases can be dispensible for migration if the cells passage through gaps and pores that are large enough to accommodate the nucleus and as long as the nucleus is able to deform. It will be important to dissect different cell types and ECM environments for the presence, necessity and consequences of proteolytic tissue remodeling.

Acknowledgements We gratefully acknowledge Dr. H. Lü for sample preparation and image acquisition. The work was supported by DFG grant FR1155/8-2 and EU a grant within the European Network of Excellence of Molecular Imaging Laboratories (EMILLSHC-CT-2004-503569) to PF.

Open Access This article is distributed under the terms of the Creative Commons Attribution Noncommercial License which permits any noncommercial use, distribution, and reproduction in any medium, provided the original author(s) and source are credited.

\section{References}

1. Lauffenburger, D. A., \& Horwitz, A. F. (1996). Cell migration: a physically integrated molecular process. Cell, 84, 359-369.

2. Kirfel, G., \& Herzog, V. (2004). Migration of epidermal keratinocytes: mechanisms, regulation, and biological significance. Protoplasma, 223(2-4), 67-78.

3. Lotz, M. M., Rabinovitz, I., \& Mercurio, A. M. (2000). Intestinal restitution: progression of actin cytoskeleton rearrangements and integrin function in a model of epithelial wound healing. American Journal of Pathology, 156(3), 985-996.

4. Friedl, P., \& Brocker, E. B. (2000). The biology of cell locomotion within three-dimensional extracellular matrix. Cellular and Molecular Life Sciences, 57(1), 41-64. 
5. Tamariz, E., \& Grinnell, F. (2002). Modulation of fibroblast morphology and adhesion during collagen matrix remodeling. Molecular Biology of the Cell, 13(11), 3915-3929.

6. Maaser, K., Wolf, K., Klein, C. E., Niggemann, B., Zanker, K. S., Brocker, E. B., et al. (1999). Functional hierarchy of simultaneously expressed adhesion receptors: integrin alpha2beta1 but not CD44 mediates MV3 melanoma cell migration and matrix reorganization within three-dimensional hyaluronan-containing collagen matrices. Molecular Biology of the Cell, 10(10), 30673079 .

7. Rhee, S., \& Grinnell, F. (2007). Fibroblast mechanics in 3D collagen matrices. Advanced Drug Delivery Reviews, 59(13), 1299-1305.

8. Petroll, W. M., \& Ma, L. (2003). Direct, dynamic assessment of cell-matrix interactions inside fibrillar collagen lattices. Cell Motility and the Cytoskeleton, 55(4), 254-264.

9. Wolf, K., Wu, Y. I., Liu, Y., Geiger, J., Tam, E., Overall, C., et al. (2007). Multi-step pericellular proteolysis controls the transition from individual to collective cancer cell invasion. Nature Cell Biology, 9, 893-904.

10. Gaggioli, C., Hooper, S., Hidalgo-Carcedo, C., Grosse, R., Marshall, J. F., Harrington, K., et al. (2007). Fibroblast-led collective invasion of carcinoma cells with differing roles for RhoGTPases in leading and following cells. Nature Cell Biology, 9(12), 1392-1400.

11. Wolf, K., \& Friedl, P. (2008). Tube travel: the role of proteases in individual and collective cancer cell invasion. Cancer Research, 68, 7247-7249.

12. Sheetz, M. P., Felsenfeld, D., Galbraith, C. G., \& Choquet, D. (1999). Cell migration as a five-step cycle. Biochemical Society Symposia, 65, 233-243.

13. Ridley, A. J., Schwartz, M. A., Burridge, K., Firtel, R. A., Ginsberg, M. H., Borisy, G., et al. (2003). Cell migration: integrating signals from front to back. Science, 302(5651), 1704-1709.
14. Miron-Mendoza, M., Seemann, J., \& Grinnell, F. (2008). Collagen fibril flow and tissue translocation coupled to fibroblast migration in 3D collagen matrices. Molecular Biology of the Cell, 19(5), 2051-2058.

15. Even-Ram, S., \& Yamada, K. M. (2005). Cell migration in 3D matrix. Current Opinion in Cell Biology, 17(5), 524-532.

16. Wolf, K., \& Friedl, P. (2008). Mapping proteolytic cancer cellextracellular matrix interfaces. Clinical \& Experimental Metastasis, Jul 4. [Epub ahead of print].

17. Friedl, P., \& Brocker, E. B. (2000). T cell migration in threedimensional extracellular matrix: guidance by polarity and sensations. Developmental Immunology, 7(2-4), 249-266.

18. Friedl, P., \& Wolf, K. (2003). Proteolytic and non-proteolytic migration in tumor cells and leukocytes. Biochemical Society Symposia, 70, 277-285.

19. Friedl, P., Borgmann, S., Brocker, E. B. (2001). Leukocyte crawling through extracellular matrix and the Dictyostelium paradigm of movement-lessons from a social amoeba. Journal of Leukocyte Biology, 70, 491-509.

20. Wolf, K., Muller, R., Borgmann, S., Brocker, E. B., \& Friedl, P. (2003). Amoeboid shape change and contact guidance: T-lymphocyte crawling through fibrillar collagen is independent of matrix remodeling by MMPs and other proteases. Blood, 102(9), 3262-3269.

21. Lammermann, T., Bader, B. L., Monkley, S. J., Worbs, T., Wedlich-Soldner, R., Hirsch, K., et al. (2008). Rapid leukocyte migration by integrin-independent flowing and squeezing. Nature, 453(7191), 51-55.

22. Friedl, P., \& Weigelin, B. (2008). Interstitial leukocyte trafficking and immune function. Nature Immunology, 9, 839-848.

23. Wolf, K., Mazo, I., Leung, H., Engelke, K., von Andrian, U. H., Deryugina, E. I., et al. (2003). Compensation mechanism in tumor cell migration: mesenchymal-amoeboid transition after blocking of pericellular proteolysis. Journal of Cell Biology, 160(2), 267277. 